\title{
Vibrations of Completely Free Rounded Regular Polygonal Plates
}

\author{
C. Y. Wang \\ Departments of Mathematics and Mechanical Engineering Michigan State University, East Lansing, MI 48824
}

(Received 28 February 2013; accepted 5 March 2014)

The vibrations of completely free polygonal and rounded polygonal plates are important for large floating or space platforms. The problem is solved by an improved Ritz method on a class of homotopy shapes. The first five frequencies are determined, and interesting evolutions of mode shapes are shown.

\section{INTRODUCTION}

Vibration of elastic plates is essential in structural mechanics. Basic data (frequencies, mode shapes) of plate vibrations can be found in the works of Leissa ${ }^{1}$ and Blevins. ${ }^{2}$ Exact solutions exist for the vibration of circular and annular plates, some simply supported triangular plates, and rectangular plates with two simply supported opposite edges. ${ }^{3}$ For all other shapes or boundary conditions numerical means are necessary.

The vibrations of regular polygonal plates have also been studied, notably Conway ${ }^{4}$ using point match, Shahady et al. ${ }^{5}$ using conformal mapping, Irie et al. ${ }^{6}$ using membrane analogy, Liew and $\mathrm{Lam}^{7}$ using a Ritz method, and Ghazi et al. ${ }^{8}$ using finite elements. These sources however, only consider simply supported or clamped edges.

We are interested in the vibration of completely free plates, i.e. all edges are free. The study is important in the design of very large ocean floating structures (e.g. ${ }^{9}$ ) and also large structures in space, such as platforms and solar panels (e.g. ${ }^{10}$ ). These structures have nominal dimensions in kilometres, much larger than their thicknesses, thus can be modelled as thin plates.

Aside from the data presented by Leissa, ${ }^{1}$ the vibrations of completely free rectangular plates includes Leissa ${ }^{11}$ using the Ritz method, Gorman ${ }^{12}$ using a superposition method, Behnke and Mertins ${ }^{13}$ using the Ritz method, and Mochida and Ilanko ${ }^{14}$ using superposition and finite differences. The free triangular plate was considered by Leissa and Jaber ${ }^{15}$ and the free trapezoidal plate by Qatu et al., ${ }^{16}$ both sources using the Ritz method. However, the natural vibration of the free regular polygonal plate has not been fully investigated.

The purpose of the present note is to study not only the vibrations of completely free regular polygonal plates, but also the more general class of rounded regular polygonal plates, which includes the polygonal plates as special cases. Rounding the corners of a polygonal plate has definite advantages in terms of savings in material, weight, and boundary length, while it changes little in the structural strength or vibration properties.

\section{THE HOMOTOPY SHAPES}

The homotopy shapes are first introduced by Wang ${ }^{17}$ and applied to the vibration of membranes. The homotopy transform

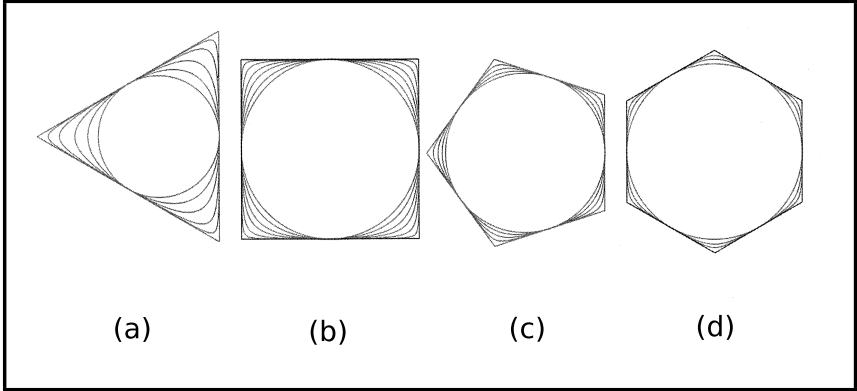

Figure 1. (a) Rounded triangular plate. From outside, $\alpha=0,0.01,0.05,0.2$, $0.5,1$. (b) Rounded square plate. From outside, $\alpha=0,0.01,0.05,0.15,0.3$, $0.6,1$. (c) Rounded pentagonal plate. From outside, $\alpha=0,0.05,0.2,0.5,1$. (d) Rounded hexagonal plate. From outside, $\alpha=0,0.05,0.2,1$.

is briefly described as follows: Let all lengths be normalized by the radius of the inscribing circle of the polygon; for a rounded equilateral triangular plate we set

$$
\begin{array}{r}
H=\alpha\left(1-x^{2}-y^{2}\right)+ \\
(1-\alpha)(1-x)\left[\left(1+\frac{x}{2}\right)^{2}-\frac{3}{4} y^{2}\right]=0
\end{array}
$$

where $\alpha=0$ is an equilateral triangle of edge length $2 \sqrt{3}$, and $\alpha=1$ is a circle of radius one. The homotopy, as $\alpha$ is increased from 0 to 1 , gives a family of rounded triangles shown in Fig. 1(a). The maximum distance from the centroid is found to be

$$
l=\frac{2}{1+\sqrt{\alpha}} .
$$

The degree of rounding is represented by the distance $d$ to the original corner

$$
d=2-l \text {. }
$$

For a rounded square plate (Fig. 1(b)) we set

$$
H=\alpha\left(1-x^{2}-y^{2}\right)+(1-\alpha)\left(1-x^{2}\right)\left(1-y^{2}\right) .
$$

The maximum distance and degree of rounding are

$$
l=\sqrt{\frac{2}{1+\sqrt{\alpha}}}, d=\sqrt{2}-l .
$$

The rounded regular pentagonal plate is shown in Fig. 1(c). 